\title{
Effect of disease modifying agents on the lipid profiles of patients with rheumatoid arthritis
}

\author{
R Munro, E Morrison, A G McDonald, J A Hunter, R Madhok, H A Capell
}

\begin{abstract}
Objective-To determine the effect of intramuscular gold and oral hydroxychloroquine(HCQ) on the lipid profile of patients with rheumatoid arthritis (RA). Method-A prospective randomised clinical trial of 12 months' duration was performed in $100 \mathrm{RA}$ patients. Data on clinical and laboratory parameters of disease activity, and fasting serum lipid samples was collected at baseline and at three monthly intervals over one year.

Results-The expected second line response was seen with no significant difference in efficacy between the groups at 12 months. The HCQ group had a significant overall improvement in their lipid profile while there was a trend for lipid profiles in the gold group to worsen.

Conclusions-HCQ is an effective second line agent that has beneficial effects on serum lipids. This should be taken into account when choosing a disease modifying anti-rheumatic drug in patients who suffer from RA and who have significant cardiovascular risk factors.
\end{abstract}

(Ann Rheum Dis 1997;56:374-377)

Rheumatoid arthritis (RA) patients die earlier than the general population ${ }^{1}$ and part of this increased mortality results from atherosclerotic coronary artery disease. ${ }^{23}$ Serum lipid concentrations are predictive for the development of atherosclerosis: men with cholesterol in the highest fifth of the population range have 3.5 times the risk of ischaemic heart disease (IHD) compared with those in the lowest fifth. ${ }^{4}$ The ratio of high density lipoprotein (HDL) to total cholesterol is an even stronger predictor of risk. $^{5}$

Patients with active RA have abnormal lipid profiles as evidenced by a global reduction of all lipid subsets and also increased triglyceride concentrations during active disease. There is some evidence that controlling disease activity may reverse some of these changes. ${ }^{6}$

We studied the effect of two disease modifying agents on lipid profiles in RA. Hydroxychloroquine (HCL) was chosen as previous reports have suggested that it may have beneficial effects on lipid metabolism independent of any changes resulting from its second line effect.

Intramuscular gold has no reported direct influence on lipid profiles and was used as a comparative agent as any changes seen could be assumed to result from changed disease activity.

\section{Methods}

PATIENTS

One hundred patients with active RA were randomised to receive either oral HCQ or injectable gold (sodium aurothiomolate) and followed up for one year. The gold dose was 50 mg weekly until response then the frequency was gradually reduced until maintenance injections were four times weekly. HCQ 400 $\mathrm{mg}$ was given for six months, then $200 \mathrm{mg}$ for six months. This reduction in HCQ dose is part of our unit's policy to try and avoid the ocular side effects that may occur with prolonged HCQ use and that are related to daily dose. Non-steroidal anti-inflammatory drugs were allowed as required; one patient in the gold group was taking oral corticosteroid. Disease duration and initial weight were recorded.

Routine blood tests for safety monitoring were performed by family doctors. Study review was at $0,3,6$, and 12 months and the following parameters were recorded; pain score $(10 \mathrm{~cm}$ visual analogue), articular index (Ritchie), erythrocyte sedimetation rate (ESR) and $\mathrm{C}$ reactive protein (CRP); lipids (measured after an overnight fast); total cholesterol, high density cholesterol (HDL), and triglycerides measured separately using previously described methods. ${ }^{7-9}$ Percentage HDL (HDL/total cholesterol $\times 100)$ was also recorded.

STATISTICAL METHODS

Analysis of within group changes were performed using a Wilcoxon rank sum test. Mann-Whitney analysis was used to examine differences between groups. The relation between lipid values and acute phase response was analysed using a Spearman correlation coefficient. All statistical calculations were performed on a personal computer using SPSS for Windows (version 6.1.1). 
Table 1 Demographic, clinical, and laboratory parameters

\begin{tabular}{|c|c|c|c|c|c|c|}
\hline & \multicolumn{3}{|l|}{ Gold } & \multicolumn{3}{|l|}{$H C Q$} \\
\hline & 0 months & 12 months & Wilcoxon & 0 months & 12 months & Wilcoxon \\
\hline Patients (n) & 49 & 25 & & 51 & 35 & \\
\hline Age (y) & $62(30-80)$ & & & $59(33-78)$ & & \\
\hline Male:female & $18: 31$ & & & $10: 41$ & & \\
\hline Disease duration (y) & $6(1-37)$ & & & $8(1-40)$ & & \\
\hline Weight $(\mathrm{kg})$ & $66.8(47.0-107.0)$ & & & $64.6(41.4-87.5)$ & & \\
\hline $\operatorname{ESR}(\mathrm{mm} / 1 \mathrm{st} \mathrm{h})$ & $63(8-130)$ & $40(3-86)$ & $\mathrm{p}=0.0013$ & $53(10-110)$ & $31(8-102)$ & $\mathrm{p}=0.0001$ \\
\hline $\mathrm{CRP}(\mathrm{IU} / 1)$ & $55(0-222)$ & $19(0-135)$ & $\mathrm{p}=0.019$ & $41(0-154)$ & $15(0-118)$ & $\mathrm{p}=0.005$ \\
\hline Pain score (VAS) & $50(10-100)$ & $45(5-100)$ & $\mathrm{p}=0.085$ & $50(0-100)$ & $38(0-95)$ & $\mathrm{p}=0.132$ \\
\hline Articular index & $12(2-42)$ & $6(0-31)$ & $\mathrm{p}=0.088$ & $12(0-42)$ & $5(0-24)$ & $\mathrm{p}=0.0001$ \\
\hline
\end{tabular}

Median and ranges are shown. No significant changes between the groups at outset (Mann-Whitney).

\section{Results}

CLINICAL

The results in table 1 show that both groups were well matched at outset (Mann-Whitney). Thirty three per cent of gold and $37 \%$ of HCQ treated patients were current smokers. Twenty five gold patients $(51 \%)$ and 35 HCQ patients $(69 \%)$ completed one year of treatment. At one year there were significant improvements in a number of parameters of disease activity in each group (table 1, Wilcoxon matched pairs); there were no significant differences between the groups (Mann-Whitney). The patient size in each group however was small and there was only a $45 \%$ chance of detecting a $50 \%$ difference in ESR reduction between the groups.

Five patients were taking thiazide diuretics (one, gold, four, HCQ). Eleven of the women were receiving hormone replacement therapy (four, gold, seven, HCQ). Table 2 gives the reasons for withdrawal from the study.

LIPIDS

Table 3 summarises the initial lipid results. There were no significant differences for total cholesterol, HDL or triglycerides at outset between those who failed to complete a year of treatment and those remaining on treatment at one year (Mann-Whitney). Percentage HDL was significantly higher in those who completed the study (23.6 $v 18.8, \mathrm{p}=0.014$, Mann-Whitney). The median values over 12 months for cholesterol, triglycerides, HDL, and $\% \mathrm{HDL}$ are shown in figure 1 for those that completed a year of treatment. Mann-Whitney $\mathrm{p}$ values for between group analysis are shown.

TOTAL CHOLESTEROL

Although total cholesterol was initially slightly lower in the HCQ group this difference was not significant. The cholesterol profiles over the 12 months were similar for both groups with there being no significant differences between the groups (Mann-Whitney) or within the groups (Wilcoxon).

Table 2 Outcome at one year

\begin{tabular}{lll}
\hline & $\begin{array}{l}\text { Gold } \\
(n=49)\end{array}$ & $\begin{array}{l}H C Q \\
(n=51)\end{array}$ \\
\hline Completed 12 months & 25 & 35 \\
Withdrew (lack of effect) & 2 & 6 \\
Withdrew (side effect) & 18 & 6 \\
Died (unrelated cause) & 2 & 0 \\
Other & 2 & 4 \\
\hline
\end{tabular}

HDL CHOLESTEROL

There was a rise in the HDL values in the HCQ group compared with a decline in the gold group (fig 1B). This reached significance at six months $(\mathrm{p}=0.034)$, which was maintained at 12 months $(p=0.040)$. Overall there was a median rise in HDL in the HCQ group of $15 \%$ compared with a fall of $12 \%$ in the gold group by 12 months. The rise in HDL in the HCQ group was significant from three months onwards $(p=0.006$ to 0.010 , Wilcoxon) although the fall seen in the gold group was only significant at six months $(\mathrm{p}=0.038$, Wilcoxon $)$.

$\%$ HDI

Figure 1C shows the \%HDL changes. At the end of the 12 month period the median \% HDL in the HCQ group had increased by $9 \%$ $(\mathrm{p}=0.040$, Wilcoxon) while that in the gold group had decreased by $15 \% \quad(p=0.062$, Wilcoxon). There was a significant difference between the groups at six months and this was maintained at 12 months $(\mathrm{p}=0.005$ to $<0.001$, Mann-Whitney).

TRIGLYCERIDES

The triglyceride concentrations increased in the gold group throughout the 12 months (31\% over the year, Wilcoxon $\mathrm{p}=0.014$ ) (fig 1D). The triglyceride concentration in the HCQ group remained virtually constant over the study period. Differences between the groups first became apparent at three months and were highly significant by 12 months ( $p=0.014$ to 0.002 , Mann-Whitney).

DISEASE ACTIVITY AND LIPIDS

There was an inverse correlation for CRP with total cholesterol at baseline $(r=-0.267$, $\mathrm{p}=0.015$ Spearman), the relation with ESR was not significant $(r=-0.177, \mathrm{p}=0.112)$. At 12 months there were no significant correlations between ESR or CRP with any of the lipid variables apart from triglycerides in the HCQ group and ESR $(r=0.450, \mathrm{p}=0.014)$. This may be a spurious result particularly as there was no significant change in the triglyceride value with treatment.

\section{Discussion}

The increase in mortality in RA equates to a median of four years of life lost in men and up to 10 years in women. ${ }^{1}$ Cardiovascular disease accounts for part of this increase in mortalitya recent Finnish study of over 1000 women with RA calculated a standard mortality ratio 
Table 3 Initial lipid profiles (by therapy by those who completed 12 months of treatment)

\begin{tabular}{lllll}
\hline & Cholesterol (mmol/l) & HDL (mmol/l) & $\% H D L$ & Triglycerides (mmol/l) \\
\hline Gold $(\mathrm{n}=25)$ & $5.41(2.66-10.10)$ & $1.22(0.85-2.65)$ & $23.5(11.9-35.6)$ & $1.16(0.55-4.30)$ \\
HCQ $(\mathrm{n}=35)$ & $5.05(3.39-7.70)$ & $1.20(0.68-2.15)$ & $24.4(11.9-49.3)$ & $1.07(0.09-3.40)$ \\
Non-completers $(\mathrm{n}=40)$ & $5.13(3.10-8.35)$ & $1.05(0.70-1.75)$ & $18.8(10.9-20.9)$ & $1.35(0.14-3.16)$ \\
\hline
\end{tabular}

Medians and ranges are shown. There were no significant differences between the gold and HCQ groups (Mann-Whitney).

of 1.51 for IHD. In that study cardiovascular disease as a whole accounted for $40 \%$ of excess mortality in the group. ${ }^{3}$

The reason for the excess mortality from IHD in RA is not clear but is probably multi-factorial. Reduced cardiovascular fitness caused by immobility and the increased incidence of RA in male cigarette smokers may be of some relevance. ${ }^{10}$ There is no evidence to implicate large or small vessel vasculitis as a cause. While serum lipids are a good predictor of IHD risk in the general population, their role in RA patients is less clear, particularly as there are alterations in lipids with changes in disease activity.

The changes in the gold group probably reflect alterations in lipid profile when active rheumatoid disease is controlled. Overall the changes seen would lead to an increase in cardiovascular risk due to a rise in triglycerides and a trend for a fall in HDL and \%HDL. The increase in triglycerides that occurs with reduced disease activity has been noted in patients treated with penicillamine. ${ }^{11}$ The above results however are in disagreement with a previous study of gold therapy that found no
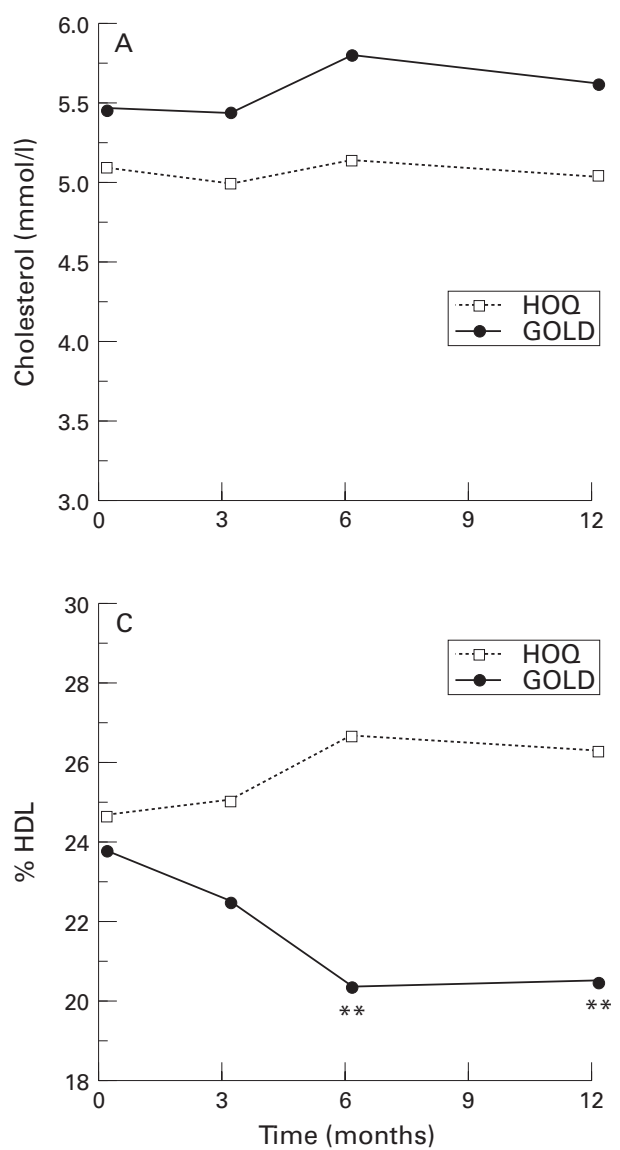

change in triglycerides and an increase in total cholesterol, LDL, and HDL values with reduced disease activity. ${ }^{6}$ The numbers involved in this conflictory study however were very small and the follow up period was only nine months.

Hydroxychloroquine may change lipid profiles in RA in one of two ways. Firstly, like gold it can lead to a reduction in disease activity by its disease modifying effect. Secondly, HCQ can change lipid metabolism directly. Animal studies have shown that chloroquine therapy leads to an overall reduction in hepatic cholesterol synthesis ${ }^{12}$ and an increase in the number of LDL receptors on fibroblasts. ${ }^{13}$

In contrast with our findings with gold therapy, treatment with HCQ led to a rise in both HDL and \%HDL values with no change in triglyceride levels. This was despite the fact that HCQ was effective in controlling disease activity. A number of previous studies have also shown HCQ has a beneficial effect on the lipid profiles of patients with rheumatic diseases. These effects have mainly been in reducing total cholesterol and LDL values, none to date has recorded the beneficial rise in HDL and
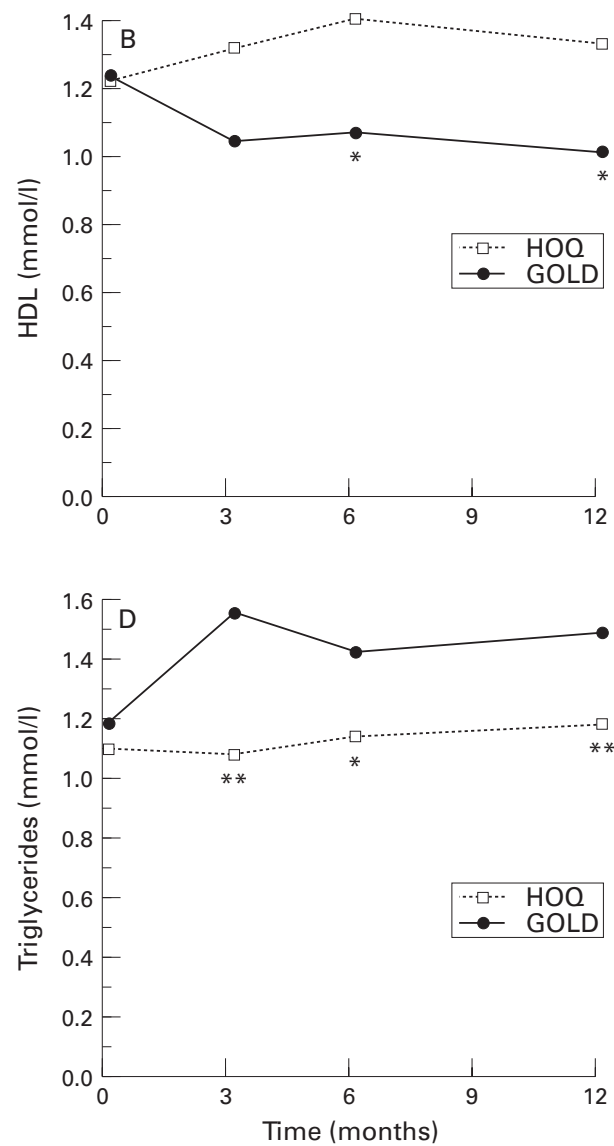

Figure 1 Median changes in (A) total cholesterol, (B) HDL, (C) \%HDL, and (D) triglycerides from baseline (between group comparisons ${ }^{*} p<0.05$ and ${ }^{\star}{ }_{p}<0.01$ Mann-Whitney). 
\%HDL noted in our study. ${ }^{14}{ }^{15}$ A possible explanation for this discrepancy is the different populations studied. Previous studies have generally included a large proportion of systemic lupus erythematosus patients many of whom were receiving oral corticosteroids. Corticosteroids themselves can lead to a rise in $\mathrm{HDL}^{16}$ and this may mask any changes caused by HCQ. Additionally most of these previous studies make no reference to changes in disease activity, which as we have mentioned can significantly influence lipid values.

A shortcoming in our study was the failure to record serial weights. The two groups were similar for weight at the outset but clearly changes may have occurred during treatment and this aspect will be considered in future studies. There was also a higher than expected drop out rate in the gold group compared with previous studies from this unit. Analysing the initial cholesterol values of those that remained on treatment compared with those who stopped treatment showed no significant differences. Thus this factor seems unlikely to explain the observed differences in the treatment groups.

There are potentially important clinical implications for the findings noted in this study. The changes that we have shown in lipids with HCQ may lead to a significant reduction in IHD risk. In populations with normal cholesterol values \%HDL (HDL/total cholesterol $\times 100)$ is a much better predictor of IHD risk than total cholesterol. A large prospective Israeli study showed that values $<23 \%$ in women and $<21 \%$ in men are associated with a threefold increase in IHD episodes compared with patients with values above these. ${ }^{17}$ Although there are few reports outlining how changes in \%HDL affect IHD risk it is probable that the magnitude of the changes seen in this study are significant. Changes in HDL values alone are known to influence IHD risk. In the Helsinki heart study using gemfibrizol an increase in HDL values was the strongest predictor of reduced cardiovascular risk. ${ }^{18}$ In that study the mean increase in HDL was $11 \%$ compared with $15 \%$ seen in the HCQ group in our study. A number of large studies suggest that there is a 2 to $2.8 \%$ reduction in IHD risk for every $0.026 \mathrm{mmol} / 1$ rise in HDL. ${ }^{4}$ In the HCQ group there is a median 0.23 $\mathrm{mmol} / \mathrm{l}$ increase in HDL, which would be expected to lead to significant reduction in cardiovascular risk.

In addition to its lipid lowering effect $\mathrm{HCQ}$ also may be cardioprotective by reducing platelet aggregation. Although we did not study platelet function this effect is pronounced enough for HCQ to have been proposed as an agent for deep vein thrombosis prophylaxis in patients undergoing hip replacements. ${ }^{19}$
In conclusion, HCQ is an effective disease modifying anti-rheumatic drug in rheumatoid arthritis. The cardioprotective properties we have shown have the potential to exert a favourable effect on the increased risk of IHD in RA patients. This should be a factor taken into consideration when prescribing a disease modifying agent for a rheumatoid patient with significant risk factors for ischaemic heart disease.

We would like to thank Mrs Marion Morrison and Mrs Anne Paterson for metrology, Mrs Dorothy McKnight for computing assistance, the Department of Biochemistry, Glasgow Royal Infirmary for lipid measurements, and Miss Ann Tierney for typing the manuscript.

1 Mitchell DM, Spitz PW, Young DY, Bloch DR, McShane DJ, Fries JF. Survival, prognosis and cause of death in heumatoid arthritis. Arthritis Rheum 1986;29:706-14

2 Prior P, Symmons DP, Scott DL, Brown R, Hawkins CF. Causes of death in rheumatoid arthritis. Br J Rheumatol 1984;23:92-9.

3 Myllykangas-Lousujarvi R, Aho K, Kautianen H, Isomaki H. Cardiovascular mortality in women with rheumatoid arthritis. J Rheumatol 1995;222:1065-7.

4 Pocock SJ, Shaper AG, Phillips AN. Concentrations of high density lipoprotein cholesterol, triglycerides and total cholesterol in ischaemic heart disease. BMJ 1989;298:9981002.

5 Grover SA, Coupal L, Xiao-Ping H. Identifying adults at increased risk of coronary disease. JAMA 1995;274:801-6.

6 Lazarevic MB, Vilic J, Mladenovic V, Myones BL, Skosey JL, Swendler WI. Dyslipoproteinemia in the course of active rheumatoid arthritis. Semin Arthritis Rheum 1992;
22:172-80.

Katterman R, Jaworek D, Möller G, Assman G, Bjorkhem I, Svensson L. Multicentre study of a new enzymatic method Svensson L. Multicentre study of a new enzymatic method
of cholesterol determination. Clin Chem Clin Biochem 1984;22:245.

8 Wahlefeld AW. Triglycerides determination after enzymatic hydrolysis. In: Bergmeyer HD, ed. Methods of enzymatic analysis. Vol 4. 2nd ed. New York: Academic, 1974:1831-5

9 Warnick GR, Albers JJ. A comprehensive evaluation of the heparin manganese precipitation procedure for estimating high density lipoprotein cholesterol. J Lipid Res 1978; 19:65-76.

10 Heliovaara M, Aho K, Aromaa A, Knekt P, Reunanen A. Smoking and risk of rheumatoid arthritis. J Rheumatol 1993;20:1830-5.

11 Svenson KL, Lithell H, Halloren R, Vessby B. Serum lipoprotein in active rheumatoid arthritis and other chronic inflammatory arthritides. II. Effects of antiinflammatory and disease modifying drug treatment. Arch Intern Med 1987;147:1917-20.

12 Benyen AC, van der Moolen AJ, Geelen MJH. Inhibition of hepatic cholesterol synthesis by chloroquine. Lipids 1981; $16: 472-4$

13 Goldstein JL, Brunschede GY, Brown MS. Inhibition of the proteolytic degeneration of low density lipoprotein in
human fibroblast by chlorquine, con A and Triton WR 1339. J Biol Chem 1975;250:7854-62.

14 Wallace DJ, Metzger AL, Stecher VJ, Turnbull BA, Kern PA. Cholesterol-lowering effect of hydroxychoroquine in patients with rheumatoid disease: reversal of deleterious effect of steroids on lipids. Am J Med 1990;89:322-6.

15 Petri M, Lakatta C, Magder L, Goldman D. Effects of prednisolone and hydroxychloroquine on coronary artery disease risk factors in systemic lupus erythematosus: a longitudinal data analysis. Am J Med 1994;96:254-9.

16 Ettinger WH, Klinefelter HF, Kwiterowich PO. Effect of short term, low dose corticosteroids on plasma lipoprotein lipids. Atherosclerosis 1987;63:167-72.

17 Brunner D, Weisport J, Mesnulam N, Schwartz S, Gross J, Saltz-Rennert H, et al. Relationship of serum total cholesterol and high-density lipoprotein cholesterol percentage to the incidence of definite coronary events: twenty year follow-up of the Donolo-Tel Aviv prospective coronary artery disease study. Am J Cardiol 1987;57:1271-6.

18 Manninen V, Elo MD, Frick MH, et al. Lipid alterations and decline in the incidence of coronary heart disease in the Helsinki heart study. JAMA 1988;260:641-51.

19 Louden JR. Hydroxychloroquine and post-operative thrombo-embolism after total hip replacement. Am J Med $1988 ; 85: 57-61$. 